\title{
Aproximación al comportamiento electoral de los centroamericanos (años 1980-1996)
}

\section{Motivación}

En marzo de 1997 se realizaron en El Salvador las segundas elecciones del nuevo régimen político instaurado a raíz de los Acuerdos de Paz de 1992. El resultado no nos tomó por sorpresa a pesar de las dificultades informativas con las cuales le dábamos seguimiento, desde la histórica ciudad de Salamanca, España, al proceso electoral. Es más, parece que se van consolidando ciertas tendencias en el comportamiento del electorado salvadoreño.

Ahora bien, la distancia "espacial" respecto de nuestro "objeto de estudio" nos ha facilitado, y en cierta forma exigido, ponerlo en una perspectiva comparad con lo que sucede en los países vecinos, me refiero al resto de países de América Central. Desde esta perspectiva ofrecemos aquí una serie de análisis exploratorios breves sobre algunas problemáticas que tienen que ver con el comportamiento electoral de los centroamericanos, los partidos y los sistemas políticos de la región y que sugieren algunas claves de interpretación de resultados electorales como los del pasado mes de marzo en el país.

\section{El problema y la metodología comparada de la ciencia política}

El comportamiento electoral, en tanto comportamiento, es un fenómeno complejo de estudiar. En él intervienen una serie de variables sin que ninguna de ellas sea determinante. El énfasis que los estudiosos han puesto en alguna o algunas variables ha tenido como resultado la formación y desarrollo de diversas escuelas o enfoques teóricos ${ }^{1}$. ¿Cuál de éstos puede semos útil para estudiar el comportamiento electoral de los centroamericanos durante los años 1980-1996? En este período, América Central se vio inmersa en una situación política de gran inestabilidad. Tres países de esta región vivieron conflictos militares internos (Guatemala, El Salvador y Nicaragua). Los procesos electorales que en estos países se llevaron a cabo quedaron así contextuados por situaciones de 
guerra civil. Desde una perspectiva geopolítica, las elecciones allí formaron parte de la estrategia contrainsurgente impulsada por Estados Unidos independientemente de si los resultados efectivos, una vez terminados los conflictos, fueron los deseados. ¿Cómo incorporamos, y cómo estudiamos, el efecto que las guerras internas han tenido sobre el electorado centroamericano y, por su medio, en instituciones democráticas como los procesos electorales y los partidos políti$\cos$ ?

Desde una perspectiva propia de la "transitología", Alcántara (1995, p. 216) ha propuesto que es importante considerar como elementos básicos en el análisis: las características del régimen político anterior, el colapso del mismo y la estrategia del cambio, entre otros. Pues bien, en Centroamérica los regímenes allí existentes antes de 1979, independientemente de su naturaleza autoritaria o democráticas, permitieron elecciones. Otra cosa es que éstas fuesen competitivas, semi-competitivas o no competitivas. La transición desde los regímenes autoritarios de Guatemala, El Salvador y Nicaragua incluyó el enfrentamiento militar. Estos son datos que hay que tener presente a la hora de interpretar las elecciones que se han dado en los regímenes recién instaurados allí. Es más, como indicábamos arriba, ni siquiera la existencia de conflictos ammados al interior de algunos de los países de la región evitaron que se realizaran eventos electorales. Si hacemos un repaso al calendario electoral centroamericano de los últimos 15 años vemos que hasta resulta paradójico que aquellos países cuyos conflictos fueron más prolongados (El Salvador y Guatemala) son los que han realizado más de lecciones. Parece pertinente pues que en este trabajo pretendamos explorar ¿cuál es el legado que, en términos de comportamiento electoral e institucionalización del sistema de partidos, han dejado las guerras a las incipientes democracias centroamericanas de Guatemala, El Salvador y Nicaragua?

El área centroamericana presenta la ventaja de abarcar cinco pequeñas naciones que comparten muchas características y difieren en otras. Por eso vamos a útilizar la comparación para resolver nuestra pregunta. De cara a nuestro objeto de estudio, vamos a aplicar una de las recomendaciones que hace Sartori para utilizar el método comparativo; comparar sistemas más semejantes porque ésto nos va a permitir "dejar de lado un alto número de variables bajo la cláusula ceteris paribus, es decir, declarándolas iguales" (1994: p.40). Desde esta perspectiva, tenemos tres países (Guatemala, El Salvador y Nicaragua) con conflictos amados intemos mientras los otros dos (Honduras y Costa Rica) no los tuvieron. Por otra parte, tenemos que los cuatro primeros países han transitado desde el autoritarismo en el período 80-96 mientras que el último tiene una democracia consolidada de medio siglo. De alguna forma, esto nos permite controlar nuestra variable independiente: la guerra. Podemos esperar que allí donde ésta estuvo presente, manteniendo todo lo demás constante, los resultados difieran en relación a donde estuvo ausente. El hecho de que Honduras haya transita- 
do sin guerra y Costa Rica sea una democracia consolidada nos puede permitir controlar los efectos de la guerra en los otros tres países, siempre y cuando hayan discrepancias significativas entre lo que se observa en los primeros y los segundos. Vamos a centramos, por la disponibilidad de información para realizar este trabajo en Salamanca, en los siguientes aspectos: participación electoral, abstención, volatilidad, fragmentación electoral y actitudes hacia el sistema político. Según el sentido de estos indicadores podemos postular hipótesis sobre las posibilidades de institucionalización de los respectivos sistemas de partidos como elemento clave para la consolidación de las democracias centroamerịcanas que surgieron luego de prolongados conflictos armados.

\section{Participación y abstención electoral}

Una de las primeras preguntas que tratan de resolver los estudios sobre el comportamiento electoral se refiere a por qué vota la gente o por qué no vota. Una primera imagen de la participación abstención en Centroamérica en los últimos 15 años nos la revela el cuadro I. Según éste tenemos un grupo de países (Honduras, Nicaragua y Costa Rica) cuya participación está arriba del $75 \%$ de los inscritos y dos países (Guatemala y El Salvador) por debajo del 65\%. Datos más conservadores bajan esos niveles de participación, al considerar a la población en edad de votar y no a los inscritos, pero se mantiene la distinción entre países (cfr. Torres-Rivas, 1992: pp.65-67). Examinemos más de cerca a cada grupo de países puesto que a primera vista pareciera que este indicador no se mueve en el sentido esperado (hacia la baja ) en Nicaragua.

Tanto en Guatemala como en El Salvador la tendencia general en la participación es a la baja. Los más altos índices de participación se registraron en dos diferentes tipos elecciones: de Asamblea Constituyente en Guatemala y de Presidente en El Salvador. Como nota curiosa, ambas elecciones ocurren el mismo año y en ambas triunfa el mismo partido: la Democracia Cristiana. Históricamente, este partido formó parte de la oposición legalizada contra los partidos gobernantes encabezados por militares. En ambos casos también, este partido aparecía como de centro acosado por partidos-agrupaciones extremistas a la izquierda y la derecha. La elección del democristiano Napoleón Duarte en El Salvador planteó claramente esta situación y recibió todo el apoyo de la administración Reagan para impulsar su estrategia contrainsurgente. Las elecciones fueron planteadas entonces como alternativa a la guerra. Resulta lógico esperar que así lo percibieran aquellos que fueron a votar.

Hacia 1984, Nicaragua también registra similares niveles de participación que Guatemala y El Salvador. Estas son las primeras elecciones desde el acceso al poder por parte de los Sandinistas en 1979. Sin embargo, son unas elecciones en donde la oposición que podía representar un desafío a los sandinistas no 


\section{Cuatro I \\ Participación electoral en América Central \\ Años 1980-1996}

\begin{tabular}{|c|c|c|c|c|}
\hline País & Año & $\begin{array}{l}\text { Tipo de elección } \\
\text { (\% inscritos) }\end{array}$ & Votantes & $\begin{array}{l}\text { Abstención } \\
\text { (\% inscritos) }\end{array}$ \\
\hline \multirow[t]{4}{*}{ Guatemala } & 1982 & Gen & 45.8 & 54.2 \\
\hline & 1984 & $\mathrm{AC}$ & 78.1 & 21.9 \\
\hline & 1985 & Gen & 69.3 & 30.7 \\
\hline & 1985 & $\operatorname{Pr}(2 a v)$ & 65.5 & 34.5 \\
\hline \multirow[t]{4}{*}{$\mathrm{Pm}=51.1$} & 1990 & Gen & 56.4 & 43.6 \\
\hline & 1991 & Ref & 15.9 & 84.1 \\
\hline & 1995 & Gen & 46.8 & 53.2 \\
\hline & 1995 & Or $(2 a v)$ & 37,0 & 63,0 \\
\hline \multirow[t]{3}{*}{ El Salvador } & 1982 & $\mathrm{AC}$ & 75.2 & 24.8 \\
\hline & 1984 & $\operatorname{Pr}(1 \mathrm{a} v)$ & 84.8 & 15.2 \\
\hline & 1985 & $\mathbf{P}$ & 61.5 & 38.5 \\
\hline \multirow[t]{5}{*}{$P m=63.7$} & 1988 & $\mathbf{P}$ & 67.7 & 32.3 \\
\hline & 1989 & Pr & 54.7 & 45.3 \\
\hline & 1991 & $\mathbf{P}$ & 51.9 & 48.1 \\
\hline & 1994 & Gen & 52,3 & 47.7 \\
\hline & 1994 & $\operatorname{Pr}(2 a v)$ & 45.5 & 54.5 \\
\hline \multirow[t]{2}{*}{ Honduras } & 1980 & $\mathrm{AC}$ & 81.4 & 18.6 \\
\hline & 1981 & Gen & 78.0 & 22.0 \\
\hline \multirow[t]{3}{*}{$\mathrm{Pm}=76.9$} & 1985 & Gen & 84.0 & 16.0 \\
\hline & 1989 & Gen & 76.0 & 24.0 \\
\hline & 1993 & Gen & 65.0 & 35.0 \\
\hline \multirow[t]{2}{*}{ Nicaragua } & 1984 & P y AC & 75.4 & 24.6 \\
\hline & 1990 & Gen & 86.2 & 13.8 \\
\hline $\mathrm{Pm}=80.5$ & 1996 & Gen & $80.0^{*}$ & $20.0^{*}$ \\
\hline \multirow[t]{2}{*}{ Costa Rica } & 1982 & Gen & 78.6 & 21.4 \\
\hline & 1986 & Gen & 81.8 & 18.2 \\
\hline \multirow[t]{2}{*}{$P m=80.9$} & 1990 & Gen & 81.8 & 18.2 \\
\hline & 1994 & Gen & 81.1 & 18.9 \\
\hline
\end{tabular}

Gen = general; $\mathrm{AC}=$ Asamblea Constituyente $\mathrm{Pr}=$ Presidencial; $\mathrm{P}=$ Parlamento; Ref = Referendo

Pm = participación medio.

Nota: Los datos de participación podrían ser menores si se considera la población en edad de votar (18 años para todos los países, excepto en Nicaragua donde es de 16 años).

* Datos aproximado tomado de ID (1996).

Fuente: FLACSO-Costa Rica, 1995; Torres-Rivas, 1992, Villacorta, 1995. 
participa. Tienen que ser las elecciones de 1990 las que efectivamente sean planteadas al electorado como alternativa a la guerra. En esas condiciones, y con la participación de todo el espectro político, en unas elecciones cuyos grandes

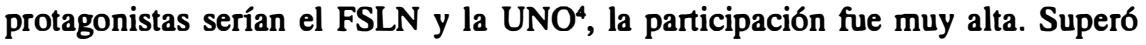
incluso los niveles observados en la democracia costarricense.

En Honduras, el comportamiento fue más errático. Ninguna elección fue planteada en oposición a la guerra puesto que ésta estaba fuera de sus fronteras. Las elecciones que registraron la mayor participación fueron las de Asamblea Constituyente y las generales de 1985. A partir de entonces, en Honduras la participación ha ido a la baja aunque no en los niveles que ocurre para los mismos años en Guatemala y El Salvador. Podemos pensar que este descenso no está asociado a la guena sino a otras causas como veremos más adelante.

Parece pues que cuando las elecciones fueron planteadas como altemativa a la guerra, por primera vez, los niveles de participación fueron muy altos en aquellos países que vivían una guerra. Al no resolverse ésta por dicho método democrático y recurrir a él frecuentemente, como en Guatemala y El Salvador, es lógico pensar que se fue profundizando una desconfianza y una falta de credibilidad, a la vez que frustración en el electorado.

Una mirada hacia atrás del período considerado en este trabajo nos da más pistas de interpretación. Por ejemplo, en Guatemala desde 1970 los niveles de participación andaban alrededor del 50\% (cfr. FLACSO-Costa Rica, 1995). Sólo las elecciones de 1984 presentan una participación arriba del 75\%. Hay que recordar que en este país desde los años 70 se combinó el fraude con la represión: "este sistema se caracteriza por hacer uso del proceso electoral y por la imposición de los gobernantes a través de fraudes electorales cada vez más evidentes y acompañado de la utilización de la represión como un verdadero método político para el mantenimiento del poder" (Solórzano, 1980: p. 24). Observando el caso guatemalteco podemos plantear que cuando las elecciones fueron percibidas como alternativa al conflicto militar registraron alta participación. Pero al no resolverse la guerra, el patrón de comportamiento participativo regresó a los niveles anteriores al período de estudio. Si en 1995 la participación estuvo en un $37 \%$ no es descabellado atribuirlo al cansancio, frustración y desconfianza en ese método que no habría cambiado nada después de 13 ocasiones, desde 1970, en las que se recurrió a él.

En El Salvador, la mayor participación desde los años 70 a la fecha ocurrió en 1972, año en que la UNO fue despojada de su triunfo ante el oficialista partido de aquel entonces PCN. No es raro que desde los 70 se fuese organizando lo que en los 80 sería el FMLN. Al plantear esto nos hemos saltado los años 82 y 84 que según el cuadro I presentaron mayor participación que 1972. Al respecto aclaramos que las cifras utilizadas para esos años provienen de una fuente distinta a la utilizada para el resto de años, porque la primera consideraba 
que los datos del censo electoral no eran fiables. ${ }^{5}$ Por otra parte, otro fraude ocurrió en 1977 en El Salvador, en donde de nuevo le fue arrebatado el triunfo a la UNO. La fórmula, elecciones con fraude y represión también se aplicó en El Salvador. No nos extraña que la participación siguiera a la baja en este país aún en las llamadas "elecciones del siglo" de 1994.

Curiosamente, en Nicaragua la participación electoral siempre fue superior a la de los países arriba considerados. Ya desde 1972 (aún con el régimen somozista) 1 os niveles de participación superaban el 70\% (cfr. FLACSO-Costa Rica, 1995). Pero sólo las elecciones del 90 llevaron este nivel arriba del 85\%, arriba incluso que en el caso costarricense. Como dijimos antes, esa elevada participación está asociada claramente al planteamiento altemativo elecciones-guerra.

Al estar hablando del comportamiento electoral en cuanto a participación se refiere, implícitamente hemos estado refiriéndonos a la abstención. Compartimos la opinión de que no hay explicaciones fáciles (Justel, 1995). Pero como lo afirmaron en su momento Seligson et al (Seligson \& Booth, 1995: pp. 177-8): "In Central America the credibility of the election process itself is very much in question. Those veho question it more are more likely to have doutbs about the legitimacy of the system of gobernment as a whole". Haber planteado las elecciones como alternativa a la guerra elevó los índices de participación, pero al no verse resuelto la guerra a pesar de las elecciones se favoreció el retomo a los bajos niveles del período previo con la diferencia que ahora el régimen político ha cambiado. La guerra activó la participación, pero elecciones frecuentes en guerra activaron la abstención. De cara a la consolidación de los nuevos regímenes una elección por analogía es clara: elecciones planteadas como salida a crisis económicas pueden generar expectativas que incrementen la participación (1988 en El Salvador, 1990 en Nicaragua) pero una vez visto que dichas crisis no se resuelven a pesar de las elecciones la abstención se vuelve a incrementar. No es aquí el momento de probar esta hipótesis.

Hasta aquí no hemos considerado la variable derecho electoral que podría pensarse tuvo su peso en los niveles de participación/abstención a través de la obligatoriedad o no del voto. Podríamos pensar que si el voto es obligatorio la participación sería mayor. Las cosas no fueron así en Centroamérica. Salvo en Nicaragua, en el resto de los países el voto es obligatorio. Pues bien, hemos visto que Nicaragua tiene mayores índices de participación frente a lo esperado para los casos guatemalteco y salvadoreño. Y si la comparamos con Costa Rica, nos veríamos obligados a asumir otra recomendación metodológica de Sartori para el uso del método comparativo: comparar sistemas más diferentes para que si se observa el mismo fenómeno, las diferencias no lo explican (Sartori, 1994: p.40). Es decir, si en Nicaragua se dan los índices de participación semejantes a los de Costa Rica, siendo que en la primera el voto no es obligatorio y si en la segunda, esta semejanza no es explicada por la diferencia (el derecho electoral). 


\section{Volatilidad electoral}

Según Gunther y Montero (1994: p. 472) podemos diferenciar dos tipos de volatilidad electoral: la volatilidad total, que se refiere a los cambios netos en las proporciones de votos de los partidos entre dos elecciones, y la volatilidad entre bloques, que se refiere a los cambios específicos de voto que traspasan divisiones de clases o de ideología. Ambas volatilidades se consideran a nivel agregado y no al nivel del elector individual. Una forma de medir la volatilidad electoral viene dada por la expresión: VE $=(\mathrm{S} \% \mathrm{VI}-\% \mathrm{Vo}) / 2$, donde $\mathrm{S}=$ sumatoria de las diferencias calculadas para todos los partidos; $\mathrm{V} 1$ = votos obtenidos en la elección presente y $\mathrm{Vo}=$ votos obtenidos en la elección pasada.

Ahora bien, los cambios en las proporciones de votos de los partidos no necesariamente reflejan movimientos de un partido a otro. Hay diversas fuentes. La tipología de votantes elaborada por G. Sani es ilustrativa al respecto (cfr. Sani, 1986: p. 5; además, Del Castillo, P. y Delgado, I, 1995): los cambios pueden deberse al comportamiento del electorado según sean estos votantes transferidos, movilizados, desmovilizados, nuevos votantes o excluidos. Para realizar un análisis de la volatilidad a este nivel se vuelve imprescindible el recurso a las encuestas de opinión y a la declaración de voto pasada y presente del elector entrevistado. Desde este punto de vista, nos es imposible realizar nuestro análisis a este nivel pues no contamos con este tipo de información.

Por otra parte, los cambios también pueden estar "del lado de la oferta" (Schedler, 1995), es decir, por la modificación de la oferta electoral debida a la aparición o desaparición de partidos, a la formación de alianzas electorales, etc. El caso de Guatemala y el de Nicaragua hacen muy complicado si no imposible el cálculo de la volatilidad porque gran parte de ella está precisamente "del lado de la oferta". El cuadro II (a) nos da una clara idea de la distorción que genera este fenómeno y por ello solamente hacemos unos comentarios sobre los datos allí mostrados sin calcular el índice respectivo.

En primer lugar saltan a la vista los altos porcentajes en la casilla correspondiente a los "otros" partidos para todas las elecciones consideradas (en 1984 es mayor que el alcanzado por cualquiera de los partidos específicamente considerados en ese año). En segundo lugar, el hecho de que en 1985, el dato se refiera sólo al PR nos impide calcular la variación respecto del año anterior puesto que allí PR particiṕ́ en alianza con PDCN. ¿Cuánto en PR en 1984? Imposible saberlo. En 1995 se nos presenta un caso semejante con UCN-DCG-PSD ya que los dos primeros participaron separados en 1990 y el PSD no participó. Lo único que puede decirse es que estos tres partidos juntos obtuvieron un 12.0 está por debajo de lo que cada uno de ellos obtuvo en 1990, y puesto que en el 12.9 hay votos al PSD, entonces podemos afirmar que ambos partidos perdieron electores (porcentualmente hablando), pero no sabemos quien perdió más. La aparición de FRG y FDNG en 1995 elevaría el índice de volatilidad pero no podemos saber 
si es en detrimento de los partidos pre-existentes, vía votantes transferidos, o si se trata de nuevos votantes. Si, de acuerdo al cuadro I, en 1995 bajo la participación respecto de 1990, el análisis llega a un callejón sin salida puesto que debemos considerar otra categoría de votantes: los desmovilizados. Por tanto, lo que parece prudente afirmar es que si en Guatemala hay altos indices de volatilidad ello se debe a lo inestable de la oferta electoral, o dicho en términos de Sartori, a la fluidez del sistema de partidos.

Algo parecido obtendríamos en el caso nicaragüense de acuerdo a los datos del cuadro II (b). Las alianzas UNO en 1990 y Alianza Liberal en 1996 nos hacen centramos en las cifras alcanzadas por el único partido relevante presente en las tres elecciones del período: el FSLN. Este partido ha ocupado el lugar de la segunda fuerza electoral en las dos últimas elecciones. Entre ellas sólo ha perdido cerca de 3 puntos porcentuales que bien podrían estar relacionadas con la caída en la participación electoral antes que con la transferencia de votos (cfr. cuadro I). Por otra parte, la desintegración de la UNO nos señala problemas de institucionalización del sistema de partidos también en Nicaragua. En otras palabras la volatilidad aquí también tiene su origen en el "lado de la oferta".

\section{Cuadro II (a)}

Guatemala: Votos obtenidos por los principales partidos (Porcentajes)

\begin{tabular}{lcccc}
\hline Partido & 1984 & $1985\left(1^{\mathrm{a} v}\right)$ & $1990\left(1^{\mathrm{a}} \mathrm{v}\right)$ & 1990 \\
\hline DCG & 21.2 & 38.6 & 17.4 & - \\
UCN & 17.8 & 20.2 & 22.7 & $\overline{-}$ \\
PAN & 9.5 & - & 17.3 & 36.5 \\
FRG & - & - & - & 22.0 \\
FDNG & - & - & - & 7.7 \\
PDCN-PR & 16.2 & $13.7^{*}$ & - & $\overline{12.9}$ \\
UCN-DCG-PSD & - & - & - & 20.9 \\
Otros & 32.9 & 27.5 & 18.5 & \\
\hline
\end{tabular}

- PR únicamente.

Fuente: Torres-Rivas (1992); Villacorta (1995).

Los casos de Honduras, Costa Rica y El Salvador resultan ser más adecuados para el cálculo de las volatilidades. Los cuadros II (c), II (d) y II (e) muestran la información pertinente y el índice de volatilidad agregada para cada par de elecciones. Honduras y Costa Rica muestran índices menores que El Salvador. Esto puede ser señal de la "congelación" de los electorados en los primeros países. 
De hecho, en ellos se habla de la existencia de un bipartidismo. Por otra parte, el caso salvadoreño es interesante porque nos sugiere claramente la existencia de dos tipos de volatilidad dominante en dos elecciones: 1988 (para la cual hemos mostrado en otro trabajo la ocurrencia de una elección crítica, cfr. Artiga-González, 1996) y 1994. La primera tendría su origen en "el lado de la demanda" (son los electores los que modifican su comportamiento) y la segunda por "el lado de la oferta" (con la entrada del FMLN a la lucha electoral). La persistencia de 3 de los principales partidos (ARENA, PDC y PCN) a lo largo de todo el período nos muestra cierta congelación de los electorados, sobre todo para el caso de ARENA, ya que los otros dos tienen su origen en los años sesenta. Los resultados de las elecciones parlamentarias y de concejos municipales de 1997 parecieran estar indicando cierta fortaleza electoral del FMLN aunque tal vez sea prematuro hablar de "encapsulamiento" de su electorado. En todo caso, El Salvador muestra claramente, por la vía del comportamiento electoral, un sistema de partidos en vías de consolidación en una situación intermedia entre Honduras y Costa Rica, por un lado, y Nicaragua y Guatemala, por el otro.

Cuadro II (b)

Nicaragua: Votos obtenidos por los principales partidos (Porcentajes)

\begin{tabular}{lccc}
\hline Partido & 1984 & 1990 & 1996 \\
\hline FSLN & 67.0 & 40.8 & 37.7 \\
Conservador Demócrata & 14.0 & $\overline{5}$ & - \\
Liberal Independiente & $\overline{-}$ & 4.7 & - \\
Otros & 10.0 & 4.5 & 11.3 \\
\hline
\end{tabular}

Fuentes: Torres-Rivas (1992); ID (1996)

Cuadro II (c)

Honduras: Votos obtenidos por los principales partidos (Porcentajes)

\begin{tabular}{llllll}
\hline Partido & 1980 & 1981 & 1985 & 1989 & 1993 \\
\hline Liberal & 48.0 & 54.0 & 51.0 & 44.3 & 53.0 \\
Nacional & 44.1 & 41.6 & 45.5 & 52.3 & 43.0 \\
Otros & 7.9 & 4.4 & 3.5 & 3.4 & 4.0 \\
Volatilidad electoral & 5 & 3.9 & 6.8 & 9.3 & \\
\hline
\end{tabular}

Fuente: Torres-Rivas (1992); FLACSO-Costa Rica (1995) y cálculos propios) 


\section{Cuadro II (d)}

Costa Rica: Votos obtenidos por los principales partidos (Porcentajes)

\begin{tabular}{lrrrr}
\hline Partido & 1982 & 1986 & 1990 & 1994 \\
\hline Liberación Nacional & 58.8 & 52.3 & 47.1 & 49.6 \\
Unid. social Cristiana & 33.6 & 46.1 & 51.5 & 47.6 \\
Otros & 7.6 & 1.6 & 1.4 & 2.8 \\
\hline Volatilidad electoral & 12.5 & 5.4 & 3.9 & \\
\hline
\end{tabular}

Fuente: Torres-Rivas (1992); Roviera (1994) y cálculos propios.

Cuadro II (c)

Nicaragua: Votos obtenidos por los principales partidos (Porcentajes)

\begin{tabular}{lccccccr}
\hline Partido & 1982 & 1984 & 1985 & 1988 & 1989 & 1991 & 1994 \\
\hline ARENA & 29.5 & 29.8 & 29.7 & 48.0 & 53.8 & 44.3 & 45.0 \\
PDC & 40.1 & 43.4 & 52.3 & 35.2 & 36.0 & 28.0 & 17.9 \\
PCN & 19.2 & 19.3 & 8.4 & 8.6 & 4.1 & 9.0 & 6.2 \\
FMLN & - & - & - & - & - & \multicolumn{1}{c}{} & 21.4 \\
Otros & 11.2 & 7.5 & 9.6 & 8.2 & 6.1 & 18.7 & 9.5 \\
\hline Volatilidad electoral & 4.8 & 12.5 & 22.5 & 11.9 & 18.3 & 26.3 & \\
\hline
\end{tabular}

Fuente: Torres-Rivas (1992); Artiga-González (1996); cálculos propios.

Ahora podemos preguntamos, ¿dónde interviene la variable guerra? Vamos a responder esta pregunta avanzando hacia otro punto de nuestro análisis: los sistemas de partidos.

\section{Estructuración del sistema de partidos}

Los altos niveles de volatilidad electoral que pueden observarse en Guatemala, El Salvador y Nicaragua, si se los compara con los encontrados para los casos de Honduras y Costa Rica, nos sugieren la fluidez y la inestabilidad de sus respectivos sistemas de partidos. Sin embargo, valgan algunos matices. No es lo mismo que en Guatemala se presenten 28 partidos, varios en coalición, o 19 
candidatos a la presidencia de la república en 1995 (cfr. Rojas, 1996) a que se presenten 9 partidos, tres de ellos en coalición, a la elección presidencial de 1994 en El Salvador. En el primero habría más fragmentación electoral que en el segundo. La prácticamente necesaria segunda vuelta tanto en Guatemala como en El Salvador resulta no sólo de la fórmula decisoria sino de lo disperso del voto. Si en Nicaragua, en ninguna de las tres elecciones presidenciales ha sido necesaria la segunda vuelta se debe en parte a la concentración del voto. No hay que pasar por alto, sin embargo, que en las dos últimas elecciones (1990 y 1996) para lograr esa concentración del voto ha tenido que constituirse una amplia alianza electoral: la UNO y la Alianza Liberal respectivamente. La rápida descomposición de la UNO luego de su instalación en el poder nos indica lo frágil de las alianzas allí y, por tanto, lo fluido del sistema. El cuadro III muestra los índices, tanto del número efectivo de partidos como de la fragmentación para los cinco países centroamericanos en sus dos últimas elecciones salvo para Nicaragua. Claramente se ve la existencia de un grupo de países bipartidistas (Honduras y Costa Rica) y otro de pluripartidistas (Guatemala y El Salvador). El caso nicaragüense puede confundir si se pasa por alto la naturaleza de la UNO en 1990: una gran coalición opositora de muchos partidos.

\section{Cuadro III}

Número efectivo de partidos y fragmentación electoral y (parmentaria) en Centroamérica

\begin{tabular}{|c|c|c|c|c|c|}
\hline País & año & & NEP & $\mathrm{F}$ & \\
\hline Guatemala & 1990 & 6.93 & (4.43) & 0.85 & (0.77) \\
\hline 1995 & 4.87 & (2.60) & 0.79 & $(0.61)$ & \\
\hline El Salvador & 1991 & 3.33 & (3.02) & 0.70 & $(0.67)$ \\
\hline 1994 & 3.52 & (3.06) & 0.72 & $(0.67)$ & \\
\hline Honduras & 1989 & 2.12 & (2.00) & $0 . \bar{n} 52$ & $(0.50)$ \\
\hline 1993 & 2.33 & (2.10) & 0.57 & $(0.52)$ & \\
\hline Nicaragua & 1990 & 2.18 & (2.05) & 0.54 & (0.51) \\
\hline Costa Rica & 1990 & 2.57 & (2.21) & 0.61 & $(0.54)$ \\
\hline 1994 & & (2.30) & & $(0.56)$ & \\
\hline
\end{tabular}

Fuente: Aleántara (1996) 
De cara a nuestra variable independiente analizada en este trabajo (la existencia de conflictos armados internos), tanto la volatilidad como los índices del cuadro III apuntan en la misma dirección: separan a los dos grupos de países que desde el principio venimos observando. Honduras y Costa Rica parecen ser los más estables y menos fragmentados. Allí los electores parecen estar "anclados". En esos dos países no hubo conflicto interno. El caso nicaragüense se desvía por la interferencia que causan las alianzas electorales pero comparte una característica con el caso salvadoreño: los partidos que antes estuvieron en la guerra se ubican como segundas fuerzas electorales (FSLN y FMLN). Las elecciones parlamentarias de 1997 en El Salvador han colocado al FMLN, una vez más, como segunda fuerza electoral. De mantenerse en esa posición, como parecen indicarlo ya las encuestas pre-electorales, será plausible la hipótesis de que ya lograron "anclarse" entre el electorado. De alguna manera, en la Centroamérica de los ochenta se habría generado un "cleavage" del cuál han salido esos partidos. Es muy temprano para evaluar esta situación pero habrá que seguirle la pista. Sobre todo si también vemos que otro partido "hijo del período de guerra" tiene predominio desde 1988: ARENA en El Salvador. No es extraño que este país esté más cerca de la consolidación de su sistema partidista. Falta por ver si, una vez que participe en la lucha electoral la URNG, muestra su fuerza como lo hicieron sus "parientes" FMLN y FSLN. La hipótesis clásica de Lipset y Rokkan sobre los "cleavages" estaría cumpliéndose así también en Guatemala. Es difícil predecir cuál será el resultado final de esta dinámica según la cual, la guerra habría jugado, en tanto fractura social, el papel de aglutinador de fuerzas y gestador de identidades sociales que desembocaron en partidos políticos. Lo cual, visto desde una perspectiva sistémica y "fría" a la vez habría sido un positivo.

Por otra parte, la alta volatilidad registrada en Guatemala y en Nicaragua durante este período podría estar indicando la falta de identificación de buena parte de los respectivos electorados con la mayor parte de los partidos políticos allí existentes ylo el recurso a una especie de "voto estratégico" por buena parte del electorado, no identificado, con una de las alternativas con posibilidades de ganar (el FSLN en Nicaragua) o en todo caso vinculado con el pasado reciente de guerra'. La continua alternancia en la presidencia guatemalteca por distintos partidos (DCG, MAS, PAN) en 10 años dice mucho al respecto. Si consideramos el descenso en la participación electoral registrado allí podemos pensar que dicha volatilidad obedece también a la desmovilización de votantes. Falta saber si esta desmovilización está asociada con la guerra o si se asocia más a la naturaleza del régimen anterior.

\section{Credibilidad y confíanza en las instituciones políticos}

Analizando el al to nivel de abstencionismo registrado en 1991 en El Salvador, Harto de Vera (1992: p. 53) lo atribuía "a que buena parte de la población 
percibió y aun sigue percibiendo que las elecciones tienen una escasa capacidad de incidencia en la realidad que los factores de poder se estarían disputando en otro terreno diferente al del espacio electoral". La situación de guerra que todavía persistía ese tipo de interpretación. Una vez superada la guerra, en 1994 la abstención seguía siendo alta. Como dijimos antes, haber propuesto las elecciones como vía para alcanzar la paz, siendo que ésta no se alcanzaría por esa vía sino por la negociación aumentó el descrédito de este procedimiento y de los partidos políticos entre el electorado. Un descrédito que es anterior a la guerra y que ya lo compartía también la población guatemalteca. Como señalaba Ramírez (1994:p.33) de cara alas elecciones de agosto del 94 en esa nación: "Si de algo está llena la vida política de Guatemala es de asonadas militares, golpes de estado, violencia y de tido ese interminable muestrario de atropellos a las libertades y a los principios democráticos que lamentablemente han cambiado en muchos países latinoamericanos. Como resultado de esa historia el noble pueblo guatemalteco muestra un alto grado de apatía y desconfianza hacia su instituciones y hacia las justas electorales". La guerra habría empeorado la situación. Habría llevado a niveles por debajo de aquellos que se observan en los países centroamericanos donde no hubo guerra aunque de todos modos es bajo tal como la muestra del cuadro IV . Esta observación nos remite a la intervención de otras variables y a la relativización del peso explicativo asignado a la guerra

\section{Cuadro IV}

Confianza en las instituciones del país (Escala de 1 a 5 de menos a mayor confianza)

\begin{tabular}{lcccccc}
\hline Institución & $\begin{array}{c}\text { Guatemala } \\
\text { Marzo/93 }\end{array}$ & $\begin{array}{c}\text { El Salvador } \\
\text { Sept./93 }\end{array}$ & $\begin{array}{c}\text { Honduras } \\
\text { Oct./93 }\end{array}$ & $\begin{array}{c}\text { Nicaragua } \\
\text { Ago./93 }\end{array}$ & $\begin{array}{c}\text { Costa Rica } \\
\text { Agos/93 }\end{array}$ \\
\hline Gob/P. Ejecutivo & 1.87 & 1.66 & 1.95 & 1.80 & 2.52 \\
Congreso/Parlamento & 1.73 & 1.60 & 1.79 & 1.87 & 2.51 \\
P. Judicial & 2.70 & 1.93 & 1.72 & 1.94 & 2.51 \\
Policía & 2.50 & 1.90 & 1.82 & 2.02 & 2.40 \\
FF.AA./Ejército & 2.90 & 1.65 & 2.03 & 1.87 & no tiene \\
Partidos políticos & 1.20 & 1.63 & 1.74 & 1.50 & 1.93 \\
Iglesia Católica & 3.50 & 2.00 & 3.24 & 3.24 & 3.24 \\
Medios de comunicación & 3.30 & 1.95 & 2.74 & 3.29 & 3.29 \\
\hline
\end{tabular}

Fuente: Rial (1995)

En primer lugar, saltan las diferencias entre Costa Rica y el resto de países aunque de todas formas, salvo la Iglesia Católica y los Medios de Comunicación, las puntuaciones generalmente se ubican abajo de el 2.52 , lo que nos 
indicaría un estado de "déficit de confianza" en las principales instituciones democráticas en Centroamérica. En segundo lugar, los partidos políticos están menos puntuados en los tres países que sufrieron guerras ${ }^{8}$. Es posible que la generación de políticos que desarrolló su actividad en los tiempos de guerra para sacar provecho personal de la situación haya llevado al descrédito generalizado no solo en los partidos sino en la política misma9. Por otra parte el hecho en que en Guatemala sea donde mas puntos reciben coincide con la fluidez del sistema de partidos que antes hemos señalado.

Hay que destacar que tanto la Iglesia Católica como los Medios de Comunicación son los que mayores niveles de confianza reciben. En cuanto a la primera, habría que preguntarse si ello se debe a peculiaridades de las sociedades tradicionales o si, en parte se debe a su papel desempeñado en la resolución de los conflictos internos o asuntos afines. Por su parte, la evolución que han tenido en los últimos años los medios de comunicación, no sólo en su complejizaciónprofesionalización sino también en el goce de una mayor autonomía respecto a los gobiernos de tumo, sobre todo en cuanto a noticieros y programas de formación de opinión se refiere, puede estar abonando a la confianza que en ellos depositan los centroamericanos. En general, puede decirse que la voz de la Iglesia y de los medios han sido más creíbles que las de las instituciones propiamente políticas. Es posible que este hecho sea otro producto, no deseado, de la guerra. El no contar con información directa vía encuestas nos impide afirmarlo categóricamente.

\section{A manera de conclusión}

Hemos hecho una incursión en parte de la vida política de los países centroamericanos para los años 1980-1996 con el objeto de encontrar el legado que la guerra dejó a sus incipientes democracias. Para controlar esta variable, hemos recurrido al análisis comparado de cinco casos de la región, tres de ellos con guerras internas. La ventaja de ser una región pequeña y de compatir una serie de problemáticas comunes nos permitió considerar constantes otras variables intervinientes, como por ejemplo: las crisis económicas y sus derivados, las presiones externas, etc. Concretamente hemos querido ver cuáles han sido los efectos de la guerra sobre el comportamiento electoral y la institucionalización de los respectivos sistemas de partidos. Llegados a este punto, dado lo complejo del objeto de estudio y la limitada información con que contabamos al redactar este trabajo, lanzamos las siguientes aproximaciones:

- El haber propuesto las elecciones como salidas a los conflictos armados, sin que eso fuera una realidad, favoreció el descrédito de dicho procedimiento, expresado sobre todo en los niveles de participación-abstención. En un principio, los electorados respondieron a tal propuesta y en todos los casos hay 
un incremento de la participación en ese momento. Posteriormente, la ineficacia percibida se traduce en un descenso de esa participación. La guerra se constituyó en otro elemento más a los que en el pasado autoritario minaban la confianza en el procedimiento electoral.

- La factura social expresada en' loś conflictos armados, que de alguna manera definieron bandos, ha desembocado en la formación de identidades partidarias aunque con diferencias de intensidad en los casos analizados. Sin darla por un hecho, la estructuración de los sistemas de partidos se ha visto así favorecida, en especial en el caso salvadoreño. Nicaragua está en una posición intermedia con un polo electoral consolidado (el FSLN) en tanto que Guatemala todavía es la que más fluidez presenta. La volatilidad electoral también apunta en la misma dirección, siendo menor en el caso salvadoreño e incalculable (aunque presumiblemente mayor) en Nicaragua y Guatemala.

- La situación de descomposición y desorden que acompañaron a los conflictos armados generó actitudes en la clase política que han sido percibidas por los centroamericanos como negativas. De allí su actual apatía e indiferencia hacia la política y los políticos y su generalizada desconfianza hacia las instituciones democráticas. Sin embargo, es cierto que estas actitudes no se derivan exclusivamente de la guerra puesto que también se encuentran en aquellos países donde no la hubo. Probablemente aquí está en juego la variable "eficacia" del sistema político, de tal manera que la apatía, indiferencia y desconfianza pueden estar en relación directa con percepción de la ineficacia del sistema para resolver los ingentes problemas, como "cuestiones no resueltas" aún, que afectan a las mayorías de esos países. Más investigación es necesaria al respecto.

\section{Referencias bibliograficas}

Alcántara, Manuel (1996). "Elecciones, electores y partidos en América Latina en la década de 1990", en América Latina Hoy, No. 13, pp. 7-16, Madrid: Seminario de Estudios Políticos sobre Latinoamérica (SEPLA) - Instituto de Estudios de Iberoamérica y Portugal, Universidad de Salamanca.

Alcántara, Manuel (1995). Gobernabilidad, crisis y cambio. México: Fondo de cultura Económica.

Artiga-González, Alvaro (1996). El Salvador: el ascenso electoral del partido ARENA y "las elecciones del siglo". (Tesis de Maestría en Ciencias Sociales). México: Facultad Latinoamericana de Ciencias Sociales.

De Vera, Fernando Harto (1992). "Los procesos electorales centroamericanos en la década de los 80". En Anfrica Latina. Hoy, No. 3 pp. 51-56. Madrid: Seminario de Estudios Políticos sobre Latinaomérica (SEPAL) - Universidad Complutense de Madrid.

Del Castillo, Pilar y Delgado, Irene (1995). Centroamérica en cifras, 1980-1992. San José, Costa Rica: Facultad Latinoamericana de Ciencias Sociales-Programa Costa Rica. 
Gunther, Richard y Montero, José (1994). "Los anclajes del partidismo: un análisis comparado del comportamiento electoral en cuatro democracias del sur de Europa", en Del Castillo, Pilar (ed). Comportamiento Político y Electoral, pp. 467-548. Madrid: Centro de Investigaciones Sociológicas.

ID (1996). Actualidad Latinoamericana , No. 29, pp. Madrid: Instituto Internacional del Desarrollo.

IUDOP (1995). Encuesta sobre el sistema político salvadoreño, San Salvador, Instituto Universitario de Opinión Pública-Universidad Centroamericana José Simeón Cañas (UCA)_.

Justel, Manuel (1995). La abstención electoral en España, 1977-1993. Madrid: Centro de Investigaciones Sociológicas.

Ramírez Víctor (1994). "Reporte electoral. Guatemala", en Boletín electoral latinoamericano, vol. XII, pp. 31-39, San José, Costa Rica: IIDH-CAPEL.

Rial, Juan (1995). "Percepciones sobre las instituciones democráticas y los medios de comunicación", en Perelli, Carina; Picado, Sonia y Zovatto, Daniel (comps.) Partidos y clase politica en América Latina en los 90 pp. 503-525.San José, Costa Rica: IIDHCAPEL.

Rojas B., Manuel (1996). "Procesos electorales recientes y representatividad de los partidos: el caso centroamericano", en América latina, Hoy, No. 13, pp. 38-44. Madrid: Seminario de Estudios Políticos sobre Latinoamérica (SEPLA) - Instituto de Estudios de Iberoamérica y Portugal, Universidad de Salamanca.

Rovira, Jorge (1994). "Reporte electoral . Costa Rica", en Boletín electoral lotinoameri- cano. Vol. XI.pp. 43-56, San José, Costa Rica: IIDH-CAPEL.

Sani, Giacomo (1986). "Los desplazamientos del electorado: anatomía del cambio", en Linz, Juan J. y Montero, José (eds). Crisis y cambio: electores y partidos en la España de los años ochenta, pp. 1-26. Madrid: Centro de Estudios Constitucionales.

Sartori, Giovanni (1994). “Comparación y método comparativo”, en Sartori, G. Morlino, L. (eds). La comparación en las ciencias sociales, pp. 29-49. Madrid: Alianza Universidad.

Schedler, Andreas (1995). "Under and overinstitutionalization: some ideal typical propositions conceming new and old party systems", en Working Paper No. 213, Notre Dame, In: the Helen Kellogg Institute for Intemational Studies-University of Notre Dame.

Selipson, Mitchell A et al (1995). "Eho votes in Central America? a comparative analysis", en Seligson, Mitchell A. Booth, John A. (eds.) Elections and Democracy in Central America revisited, pp. 151-182. Chapel Hill and London: The University of North Carolina Press.

Solórzano, Mario (1980). "El papel de la Democracia Cristiana en la actual coyuntura Centroamericana", en Nueva Sociedad No. 48, pp. 22-33. Caracas: Editorial Nueva Sociedad.

Torres-Rivas, Edelberto (1992). "Escenerios y lecciones de las elecciones centroamericanas (1980-1991)", en Revista Mexicana de sociologia 3/92, pp. 45-67. México: Instituto de Investigaciones sociales-UNAM.

Villacorta, Manuel (1995). "Reporte electoral. Guatemala", en Boletín electoral latinoamericano, Vol. XTV. pp. 17-30. San José, Costa rica: IIDH-CAPEL. 


\section{Notas}

1. La literatura al respecto nos revela puntos de partida diversos, metodologías específicas, información y datos de distinta clase. De acuerdo al tipo de variable que juega el papel central podemos hacer la siguiente clasificación: enfoque centrados en variables estructurales, enfoques centrados en variables psicológico-afectivas y enfoques centrados en la racionalidad de los actores, cfr. Artiga-González, 1996.

2. Restringimos, por razones históricas, la identificación de América Central a los países: Guatemala, El Salvador, Honduras, Nicaragua y Costa Rica. Esto no quiere decir que neguemos la calidad centroamericana a Belice y Panamá.

3. En el período 1980-1996, se realizaron 11 elecciones en Guatemala, 9 en El Salvador, 5 en Honduras, 4 en Nicaragua y 4 en Costa Rica. Se incluyen elecciones generales, presidenciales (1a. y 2a. vuelta), parlamentarias, municipales, de Asamblea Constituyente y referendos Cfr. Rojas, 1996; Ramírez, 1994.

4. En dichas elecciones, la UNO obtuvo el $54.7 \%$ de los votos válidos mientras que el FSLN alcanzó el $40.8 \%$.

5. Nos referimos a la base de datos de FLACSO-Costa Rica, 1995. Hemos utilizado en su defecto a Torres-Rivas, 1992. Análisis locales también eran de la idea de que los resultados electorales estaban inflados, aunque no en sus proporciones, para dar una imagen de gran apoyo a la salida electoral al conflicto armado salvadoreño, cfr. los números de la revista ECA de 1982 y 1984 dedicados al análisis de estas elecciones.

6. Si con estos datos la calculásemos para $1985-18.6 ; 1990=43.9$ y $1995=63.0$.

7. Es curioso que en los tres países en que hubo guerra gobiemen partidos o coaliciones “de la misma familia": PAN, ARENA y Alianza Liberal. Como lo señaló en su oportunidad Torres-Rivas (1992; p. 57): “En situaciones de crisis social prolongada, el voto, la elección, está determinado por consideraciones personales en las que priva la desesperación inmediata. Frente al caos social se vota por la seguridad y el orden... el discurso de la derecha tiene condiciones de ser más coherente por ser más elemental y porque tiene el atractivo de la condena al desorden (la revolución) que la izquierda promueve".

8. Una encuesta más reciente en El Salvador (Octubre/95) mostraba cómo ante la pregunta de si los políticos cumplen lo prometido, $69.6 \%$ contestabanque "nunca" y otro 26.8\% que "algunas veces" (cfr. IUDOP, 1995).

9. La misma encuesta de la nota 9 revel6 que $43 \%$ de los encuestados no se interesaban por la política, $37.6 \%$ se interesaban poco y sólo un $11.9 \%$ se interesaban mucho. 